\title{
PRELIMINARY RESULTS OF THE DETERMINATION OF ABSOLUTE PROPER MOTIONS OF STARS REFERRED TO GALAXIES
}

\author{
N.V. FATČIHIN
}

Absolute proper motions have been determined for 14000 stars in the 82 areas indicated in Figure 1. These proper motions result from measures made on 105 plate pairs taken with the Pulkovo normal astrograph which has a focal length of $3.5 \mathrm{~m}$. The plates, which had an average epoch difference of 22.4 years, were measured film-to-film in a Repsold measuring machine. The number of galaxies used for defining zero proper motion was 271 , and among the stars measured were 1283 from the AGK3. The probable error of a determination of proper motion from one plate pair was

$$
\begin{aligned}
& \pm 0.0052 \text { (reference stars) } \\
& \pm 0.007 \text { (AGK3 stars) } \\
& \pm 0.0062 \text { (galaxies). }
\end{aligned}
$$

The images of the galaxies were estimated as having an average quality of 6 on a scale of 10. (Deutsch et al., 1955).

The coordinates of the solar apex and the secular parallax as determined by the Kovalsky-Airy method using the derived proper motion for stars between photographic magnitudes $14 \cdot 5$ and $15 \cdot 0$, but omitting all those for which $\mu>0$ ".05, were

$$
\begin{gathered}
A=+302^{\circ} .5 \pm 4.5 \\
D=+32.6 \pm 2.7 \\
(\bar{h} / \rho)=0.0096 \pm 0.0006
\end{gathered}
$$

The variation of the secular parallax with galactic latitude is set out in Table 1. Since only stars within a narrow range of magnitude were considered, this variation is cosmical in origin, e.g. due to a larger percentage of giants in low galactic latitude than in high.

The proper motions of the AGK3 stars on the FK3 and FK4 systems were very kindly provided by Professor Haffner and Dr. Dieckvoss before publication. This permitted a comparison to be made between these systems and that defined by the galaxies. This comparison was limited to those AGK3 stars which are located within $40^{\prime}$ of the optical centre of the measured plates. There were altogether 742 such stars, an average of 9 stars per area.

Perek (ed.), Highlights of Astronomy, 297-300. C I.A.U. 


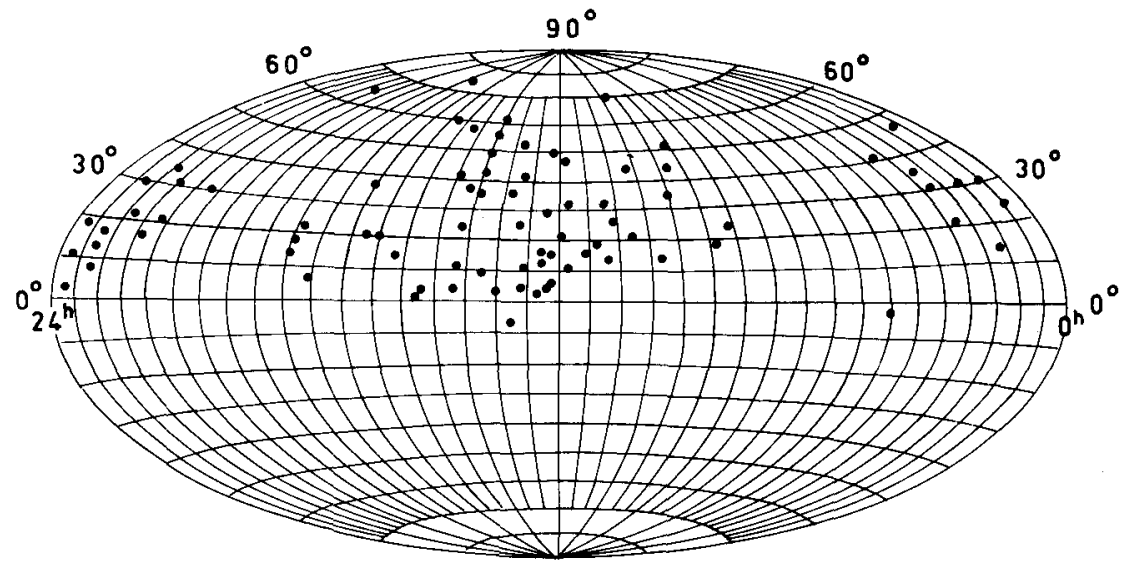

FIG. 1. The distribution of the investigated areas.

Table 1

Variations of the secular parallax with galactic latitude

Galactic latitude

From $\pm 11^{\circ}$ to $\pm 30^{\circ}$

From $+30^{\circ}$ to $\pm 50^{\circ}$

From $\pm 50^{\circ}$ to $\pm 90^{\circ}$ $\left(\begin{array}{l}\bar{h} \\ \bar{\rho}\end{array}\right)$

$$
\begin{array}{r}
+0 " 0047 \\
+0 " 0083 \\
+0 " 0112
\end{array}
$$

Probable error

Number of areas

For the determination of the correction to Newcomb's constant of precession the differences $\Delta \mu=\mu_{\mathrm{AGK} 3}-\mu_{\mathrm{G}}$ were represented by the formulae

It was found that

$$
\begin{aligned}
& \Delta \mu_{x}=\Delta m \cos \delta+\Delta n \sin \alpha \sin \delta \\
& \Delta \mu_{y}=\Delta n \cos \alpha .
\end{aligned}
$$

$$
\begin{aligned}
\Delta m & =+0.0053 \pm 0.0011 \\
\Delta n & =+0.0044 \pm 0.0011,
\end{aligned}
$$

or

where $\Delta E=\Delta \lambda+\Delta e$.

$$
\begin{aligned}
\Delta p_{1} & =+0.0111 \pm 0.0011 \\
\Delta E & =+0.0049 \pm 0.0004,
\end{aligned}
$$

The derived value of $\Delta p_{1}$ is in good agreement with other determinations. The value of $\Delta E$, on the other hand, is smaller by a factor of about 2 . The correction $\Delta e$ obtained by previous authors must therefore be explained mainly (but not completely) by either the existence of unknown systematic motions of the stars in addition to 


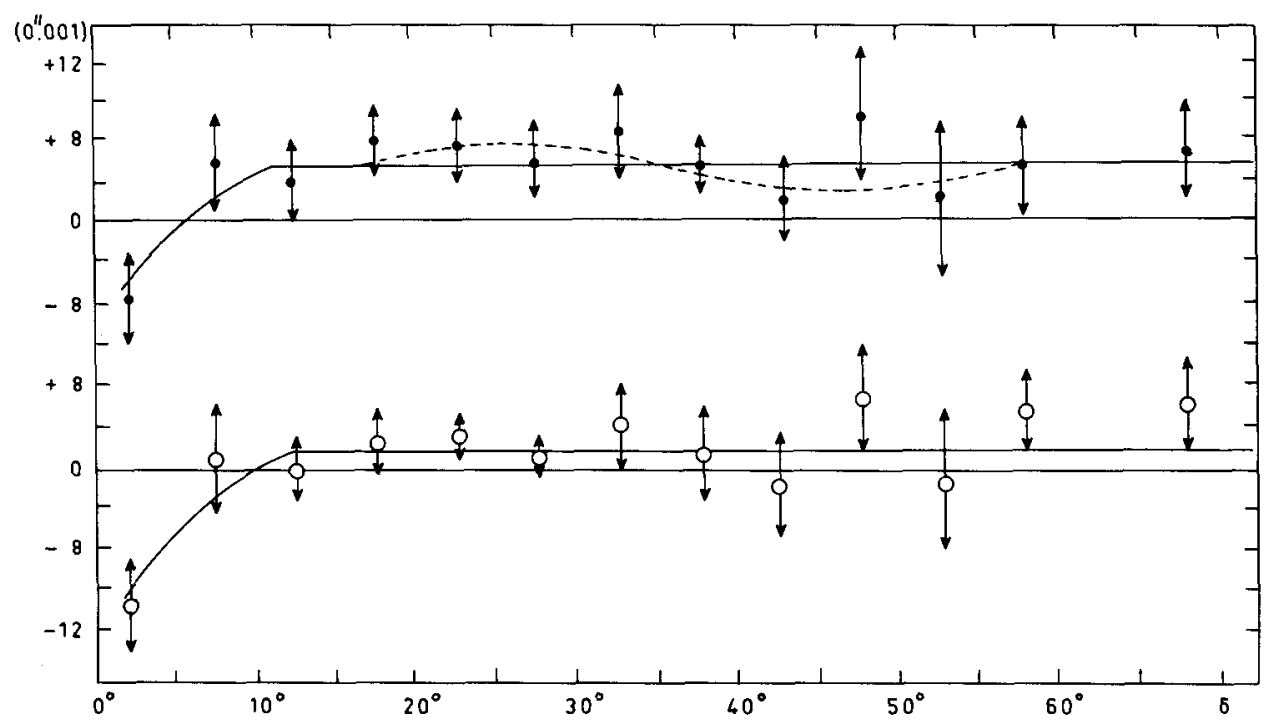

FIG. 2. The systematic differences $\left(\Delta \mu_{\mathbf{x}}\right)_{\delta}$. Top and bottom - without and with allowance for the correction to Newcomb's precessional constant.

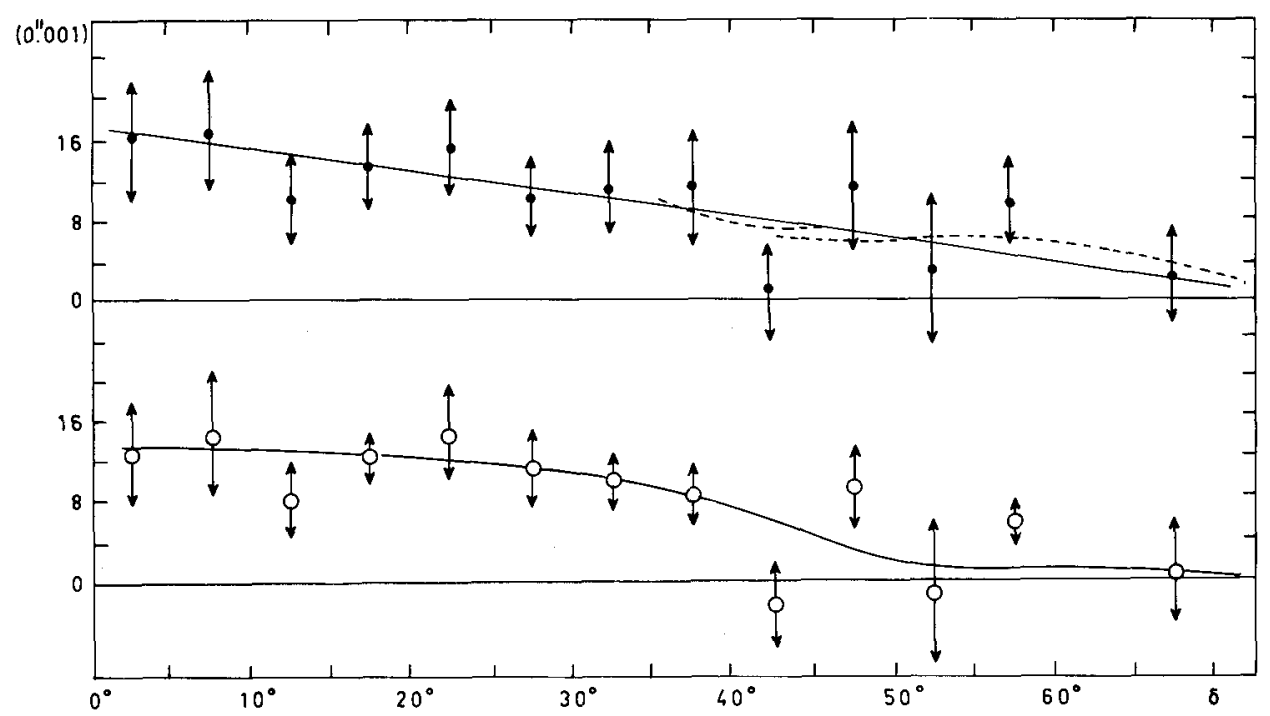

Fig. 3. The systematic differences $\left(\Delta \mu_{y}\right)_{\delta}$. Top and bottom - without and with allowance for the correction to Newcomb's precessional constant.

those due to solar motion and galactic rotation, or by an incorrect allowance for these effects. In our computations the effects of systematic motions of the stars are automatically eliminated since we are working with the differences $\mu_{\mathrm{AGK} 3}-\mu_{\mathrm{G}}$.

If it is adopted from theoretical considerations that $\Delta \lambda=0^{\prime \prime} .0008$, we will have that 


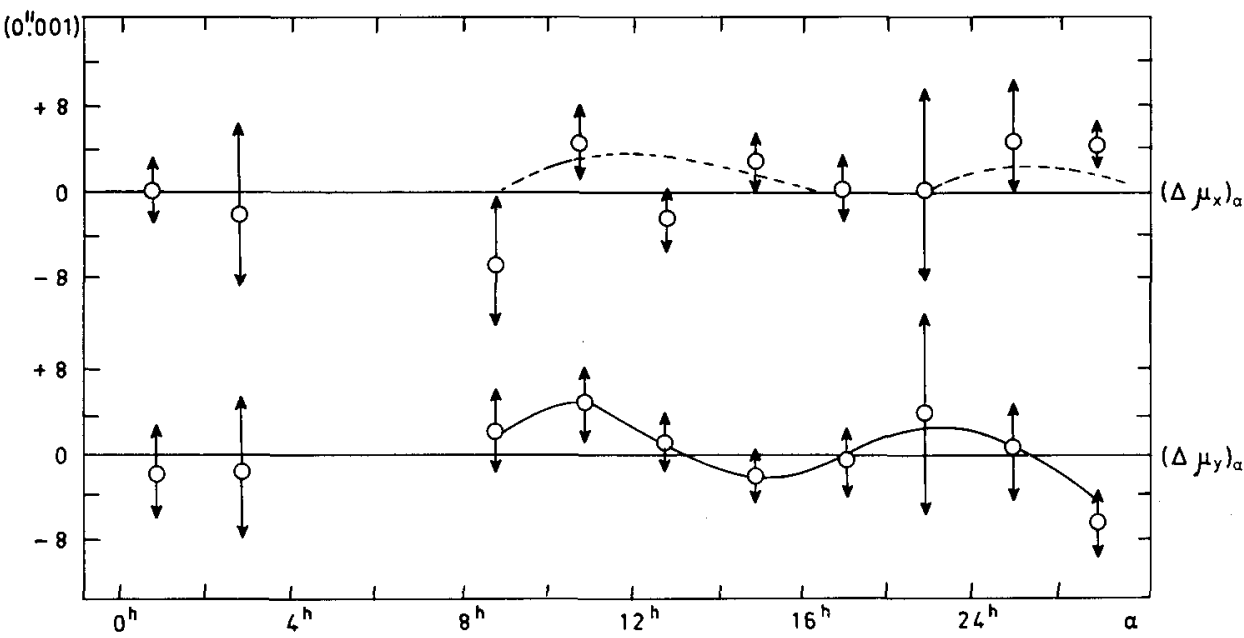

FIG. 4. The systematic differences $\left(\Delta \mu_{\mathrm{x}}\right)_{\alpha}$ and $\left(\Delta \mu_{y}\right)_{\alpha}$ with allowance for the correction to Newcomb's precessional constant.

$\Delta e=+0^{\prime \prime} .0041$. This residual is due to errors in the meridian observations of the stars, planets, and the Sun.

The variations in the systematic differences $\left(\Delta \mu_{x}\right)_{\delta},\left(\Delta \mu_{y}\right)_{\delta},\left(\Delta \mu_{x}\right)_{\alpha}$ and $\left(\Delta \mu_{y}\right)_{\alpha}$ without and with an allowance for the correction to Newcomb's precessional constant, are set out in Figures 2, 3 and 4. The allowance for the correction does not noticeably change the differences $\left(\Delta \mu_{x}\right)_{\alpha}$ and $\left(\Delta \mu_{y}\right)_{\alpha}$. The curves of the differences $\left(\Delta \mu_{x}\right)_{\alpha}$ in the declination zones $0^{\circ}-+25^{\circ}$ and $+25^{\circ}-+50^{\circ}$ are practically parallel and similar to the curves in Figure 4.

The systematic differences shown in Figures 2, 3 and 4 are to be attributed to errors in the system of AGK3 since there should be no systematic errors in the proper motions referred to galaxies. It must be emphasised, however, that the present investigation is a preliminary one and its conclusions tentative.

\section{Reference}

Deutsch, A. N., Lavdovskij, V.V., Fatčihin, N.V. (1955) Izv. Pulkovo, 154, 14. 